\title{
Cannabis use and cannabis use disorders and their relationship to mental disorders: A 10-year prospective-longitudinal community study in adolescents
}

\author{
Hans-Ulrich Wittchen ${ }^{\text {a,b,*, }}$, Christine Fröhlich ${ }^{\text {a }}$, Silke Behrendt ${ }^{\text {a }}$, Agnes Günther ${ }^{\text {a }}$, Jürgen \\ Rehm ${ }^{\mathrm{a}, \mathrm{c}}$, Petra Zimmermann ${ }^{\mathrm{a}}$, Roselind Lieb ${ }^{\mathrm{b}, \mathrm{d}}$, Axel Perkonigg ${ }^{\mathrm{a}, \mathrm{b}}$ \\ a Clinical Psychology and Psychotherapy, Technical University Dresden, D-01187 Dresden, Germany \\ b Max Planck Institute of Psychiatry, Clinical Psychology and Epidemiology, 80804 Munich, \\ Germany \\ c Centre for Addiction and Mental Health, University of Toronto, Toronto, Ontario, Canada M5S 251 \\ d Epidemiology and Health Psychology, Institute of Psychology, University of Basel, CH-4055 Basel, \\ Switzerland
}

\begin{abstract}
Background: Whereas the role of externalizing disorders is relatively well established in predicting the onset of cannabis use (CU) or cannabis use disorder (CUD), the status of anxiety and mood disorders in predicting $\mathrm{CU}$ and CUD remains controversial.

Objective: (1) To examine cross-sectional and prospective associations of CU and CUD with a range of mental disorders and whether anxiety and mood disorders are associated with CU/CUD after adjusting for externalizing disorders.

Methods: $\mathrm{N}=1395$ community subjects aged $14-17$ at baseline were followed-up at three waves prospectively over 10 years. Substance use, substance disorders and mental disorders were assessed using the DSM-IV/M-CIDI.

Results: (1) The baseline prevalence rates where $19.3 \%$ at to for CU and $2.6 \%$ for CUD. Cumulative incidence rates at $\mathrm{t} 3$ were $54.3 \%$ for CU and $13.7 \%$ for CUD. (2) In cross-sectional and prospective analyses other substance use disorders, mood and anxiety disorders were associated with CU and CUD. (3) Associations of panic-anxiety with CU and of depressive and bipolar disorders with CU and CUD were significant after controlling for externalizing disorders.

Conclusion: A range of psychopathological conditions, including depressive, bipolar and less consistently anxiety disorders as well as the degree of their comorbidity are significantly associated with incident CU and progression to CUD, even when controlling for externalising disorders. A better understanding of this complex interplay may result in better aetiological models and intervention strategies.
\end{abstract}

Keywords: Substance use disorder; Cannabis; Externalising disorders; Anxiety; Depression; Epidemiology

\section{Introduction}

A substantial number of studies have examined the relationship of mental disorders and illicit substance use and substance use disorders including cannabis. We identified six general population or community studies in adolescents (up to 18 years of age) with a prospectivelongitudinal design and standardized assessment of disorders according to DSM-IV or ICD-10 criteria that also provide data on the association of cannabis use $(\mathrm{CU}) /$ cannabis use disorder (CUD) and mental disorders (Table 1). 
The studies, however, differ considerably with regard to the coverage of diagnoses and respectively the assessment of mental disorders and syndromes, sampling, age of inclusion, length and frequency of follow-up, as well as many technical aspects of analyses, such as control for confounders. Possibly as the result of this methodological variation, there is also some variation in findings. In particular two issues remain unclear: (a) the role of anxiety and depressive (so-called internalizing disorders) for onset of CU and CUDs, (b) the role of specific disorders (such as bipolar disorders, panic disorder, social phobia, etc.), and (c) whether anxiety and depressive disorders play a role beyond the well documented effect of externalizing disorders such as conduct, anti-social personality disorder (ASP) and attentiondeficit/hyperactivity disorder (ADHD).

McGee et al. (2000) reported from the Dunedin Multidisciplinary Health and Development Study only associations of any mental disorder and CU. The other epidemiological prospective studies focused on specific disorders or major diagnostic subgroups; of these, five studies reported associations with externalizing disorders, and five with depressive and anxiety disorders. All studies that focused on externalising disorders reported consistently strong associations of conduct disorder on CU or CUD (Boyle et al., 1992; King et al., 2004; Fergusson et al., 2007; Pedersen et al., 2001; Sung et al., 2004). Boyle et al. (1992) found no association to ADHD, and King et al. (2004), in a study on twins, found only weak associations. Fergusson et al. (2007) reported that the association of ADHD and substance use disorders was mediated by conduct problems. Findings on depressive disorders are inconsistent. Overall, only weak associations are found for depressive disorders and none of these studies identified associations to hypomania/mania. Fergusson et al. (2002) found that major depression was associated with CU in the Christchurch Health and Development Study across all age groups. Boyle et al. (1992) as well found no association with depressive disorders, and King et al. (2004) reported only weak effects. In the Children in the Community Study (Brook et al., 2002) an opposite association was found, as cannabis and other illicit drug use predicted major depression. Even less attention is paid so far to the association of CU and anxiety disorders. In the Christchurch Health and Development Study (Goodwin et al., 2004) anxiety as a major diagnostic subgroup was significantly associated with substance dependence (OR: 1.3-3.9); however, after adjustment for childhood and family factors, prior substance dependence, co-morbid depression and peer affiliations, associations between anxiety and substance dependence disappeared. Associations for specific anxiety disorders remained unclear, due to low statistical power and the observation that the influence of other third variables might actually account for some or all of the association (Fig. 1).

In contrast, within the Munich Early Developmental Stages of Psychopathology (EDSP) Study various publications have highlighted significant association of substance use (SU) and substance use disorders (SUD) and specific anxiety and depressive disorders. For example, ecstasy use was associated with increased risk for almost all mental disorders examined (Lieb et al., 2002); the onset of mental disorders occurred prior to the first use of ecstasy in the vast majority of these cases. One to two year follow-up data suggested that $22 \%$ of regular CUDs had pre-existing anxiety disorders and $37 \%$ pre-existing affective disorders (Höfler et al., 1999). Wittchen et al. (2004) highlighted the increased rates of anxiety and/or depressive or childhood disorder among EDSP respondents reporting CU during adolescence or young adulthood using 4-year follow-up data and suggested that anxiety and mood disorders among CU subjects might increase the probability of progression to more severe substance use disorders. However, using the same data set, anxiety disorders were not confirmed to significantly predict progression from CU to cannabis use disorders in the 4-year follow-up (von Sydow et al., 2002a). Other noteworthy findings from their study also highlighted the 
potential critical role of CU in adolescence as a promoter for other psychopathology. For example, Henquet et al. (2005) found that CU increased the risk of psychotic symptoms especially among those with a pre-existing liability for psychosis (Henquet et al., 2005).

To summarize, few prospective-longitudinal community studies have simultaneously examined the role of various mental disorders for using cannabis and other illicit drugs, and for developing substance use disorders through adolescence and early adulthood. Based on cross-sectional and longitudinal evidence various types of externalizing disorders seem to be well established as risk factors at least for initiation of CU and the use of other substances. The role and the implications of specific anxiety, mood and other internalizing disorders in predicting cannabis and other illicit drug use disorders, however, remains controversial.

This leads to the specific objectives of this paper: (1) Because this is the first publication that includes the 10-year follow-up data we first present baseline and 10-year cumulative incidence rates of substance use and substance use disorders in adolescents aged 14-17 years at the outset of the study. (2) Focusing on cannabis as the most frequent illegal drug of abuse in this sample, we then examine cross-sectional and prospective associations of cannabis use and cannabis use disorders with a wide range of mental disorders. (3) Last, we examine whether anxiety and depressive disorders predict onset of CU and CUD beyond the effects of conduct and other externalizing disorders.

\section{Methods}

\subsection{Sample and overall design}

Data were collected as part of the Early Developmental Stages of Psychopathology (EDSP) study. Objective, sample and design have already been reported elsewhere (Lieb et al., 2000; Wittchen et al., 1998a,b). Briefly, the EDSP is designed to explore prevalence and incidence, familial and other risk factors, co-morbidity and course of substance use and substance use disorders in a representative population sample of adolescents and young adults over a 10year period with three follow-up examinations. The baseline sample was drawn in 1994 from government registries in metropolitan Munich, Germany. For the purpose of this paper only data from the four youngest age cohorts $(\mathrm{N}=1395)$, namely ages $14-17$ at baseline will be considered.

The baseline survey was conducted in $1995(\mathrm{~T} 0, \mathrm{~N}=1395$, response rate $74.6 \%)$; the followup examinations took place approximately $1.5(\mathrm{t} 1), 4(\mathrm{t} 2)$ and $10(\mathrm{t} 3)$ years later. Response rates at $\mathrm{t} 1$ for the sample considered for this publication were $88.0 \%(\mathrm{~N}=1228), 83.8 \%$ at $\mathrm{t} 2$ $(\mathrm{N}=1169)$ and $73.0 \%$ at $\mathrm{t} 3(\mathrm{~N}=1019)$. At the final wave the age range of subjects was $21-27$. It should be noted that $(\mathrm{n}=85)$ participants who denied the mandatory commitment probes to answer questions on illicit drug use and disorders truthfully and openly at one or more waves, were excluded from the analyses referring to illicit substances resulting in $\mathrm{N}=1324$ subject participants at baseline, and $\mathrm{N}=1310$ in the longitudinal sample with last case carried forward analyses.

\subsection{Diagnostic assessment}

Participants were interviewed at each wave with the baseline and respective follow-up versions of the Munich-Composite International Diagnostic Interview (DIA-X/M-CIDI) (Wittchen et al., 1995; Wittchen and Pfister, 1997), an updated version of the World Health Organization (WHO) CIDI (Wittchen and Semler, 1991). The DIA-X/M-CIDI allows for the 
assessment of symptoms, syndromes, and diagnosis of 48 mental disorders, along with information about onset, duration, severity, and psychosocial impairment. The diagnosis of mental disorders presented in this article is based on the M-CIDI/DSM-IV algorithms. The DIA-X/M-CIDI is supplemented by a separate respondents' booklet that includes disorderspecific questionnaires as well as symptom lists and cognitive aids to assist the respondent in dating symptom onset and recency, answering complicated symptom questions, and identifying course patterns. Test-retest reliability and validity for the full DIA-X/M-CIDI have been reported elsewhere (Reed et al., 1998; Wittchen, 1994; Wittchen et al., 1998a,b), along with descriptions of the DIA-X/M-CIDI format and coding conventions. A total of 32 clinical interviewers conducted the M-CIDI-interviews. All interviewers, most of whom were clinical psychologists, received an intensive 1-week training on the M-CIDI, followed by at least 10 closely monitored practice interviews.

\subsubsection{Assessment of substance use and substance use disorders.}

Use of nicotine, alcohol and drugs, including cannabis, were assessed with the M-CIDI substance use modules as well as abuse, and dependence according to DSM-IV. Details have been presented in Lachner et al. (1998), as well as von Sydow et al. (2001, 2002a,b), and Zimmermann et al. (2005). The substance use sections start with an extensive quantityfrequency screen for use of the substances followed by questions on frequency and quantity for the respective reference periods (current, time period between interviews and lifetime), as well as symptoms of abuse and dependence. All sections close with questions on onset, duration, and recency of use and symptoms. In the drug section a list containing specific substances together with their "street-names" is presented probing for eight classes: cannabis, stimulants/amphetamines, opioids, cocaine, PCP, hallucinogens, inhalants as well as sedatives/hypnotics/anxiolytics. An open category of any other substance and a category of polysubstance use were added. Questions on symptoms of DSM-IV abuse and dependence of illicit substances were only applied to those who had used these substances more than four times. Among participants unwilling to respond to illicit drug use openly ('commitment probe'), the section was not administered. Test-retest reliability of these sections ranges between a $\kappa$ of 0.55 for drug abuse and a $\kappa$ of 0.64 for nicotine dependence. Good agreement was found for the quantity and frequency questions. In case of validity there was a good agreement between DSM-IV diagnoses for substance use disorders assigned by clinicians and those assessed with the M-CIDI and assigned according to the M-CIDI DSM-IV algorithms $(\kappa=0.86)$.

\subsubsection{Assessment of mental disorders.}

Mental disorders were assessed with the M-CIDI at baseline and follow-ups using DSM-IV criteria, covering mood disorders (major depression, dysthymia, hypomania and mania/bipolar I or bipolar II), anxiety disorders (panic disorder, generalized anxiety disorder (GAD), agoraphobia, social phobia, specific phobia, separation anxiety disorder, obsessivecompulsive disorder (OCD), post-traumatic stress disorder (PTSD), somatoform disorders (pain disorder and somatization), and eating disorders. From a wide range of childhood disorders (conduct problems, ADHD, ODD (oppositional-defiant disorder)) and other childhood conditions (e.g. tics, enuresis and encopresis, sleep disorder), the externalizing disorders (conduct problems, ODD, ADHD)were used for the predictor analysis. Conduct problems were defined dimensionally with one symptom (low), two symptoms (middle), and three or more symptoms (high) out of 23 questions. Separation anxiety was assessed at first follow-up according to DSM-IV.

In the analyses "any mental disorder" was defined as having any mood disorder, any anxiety disorder (like defined above), any somatoform disorder, any substance use disorder (without 
cannabis and without all illicit substances) as well as childhood disorders. Panic-attacks and panic-disorder were aggregated into the variable panic-anxiety, to increase statistical power.

\subsection{Statistical analysis}

Data were weighted to account for different sampling probabilities (14 and 15 years olds were sampled with a higher probability) as well as systematic nonresponse at baseline (Lieb et al., 2000). The Stata Software package (StataCorp., 2003) was used to calculate proportions and standard errors as well as robust confidence intervals required when analyses are based on weighted data (Royall, 1986).

Cumulative lifetime incidences were generated using the last observation carried forward (LOCF) method, which means for each individual, missing values are replaced by the last observed value for each variable. Logistic regression models (Agresti, 1990; Long and Freese, 2003) were used to examine associations between mental disorders and CU/CUD. Prospective associations were calculated between baseline mental disorders and cumulative lifetime incidence rates for CU and CUD up to 3 . All regressions were controlled for gender. The estimated coefficients were transformed to odds ratios (ORs). Odds ratios describe the change in the chance of appearance of a particular outcome relative to the comparison group per unit increase in the covariate.

Furthermore, survival analyses were conducted in order to use the full information of onset of CU and CUDs, respectively, and prior mental disorders until T0, T1, T2, T3, taking into account the ages of onset as well as age censoring at T0, T1, T2 or T3. Cox regressions with time dependent covariates were fitted (Therneau and Grambsch, 2000) stratified for gender and age cohort. Minor numbers of missing values of age of onset data $(<10 \%)$ were replaced with the median in the respective age group (four different age groups were used). The Kaplan-Meier estimator was used to graph the age dependent cumulative lifetime incidence. Differences between cases, for example with and without a prior anxiety disorder were quantified with hazard ratios, that is, the factor by which cases and non-cases differ in the risk for the subsequent outcome. The proportional hazards assumption was tested using Schoenfeld residuals (Grambsch and Therneau, 1994).

\section{Results}

\subsection{Prevalence and cumulative incidence of substance use and substance use disorders}

Table 2 presents the baseline prevalence ( $\mathrm{t} 0)$ and 10 years follow-up ( $\mathrm{t} 3$ ) cumulative incidence of substance use (SU) and substance use disorders (SUD; abuse and dependence) of alcohol, nicotine, cannabis, and other illicit substances by baseline age group.

\subsubsection{Baseline findings for SU.}

About $90.5 \%$ of all adolescents reported having ever used any substance, most frequently alcohol $(85.7 \%)$ and nicotine $(68.6 \%)$, less frequently cannabis $(19.3 \%)$ and other illicit drugs $(4.8 \%)$. Use rates increase by age cohort, with 14 years old having the lowest use rates and 17 years old the highest. For CU, rates were 5.4\% among 14 years old, 20.8\% in 15 years old, $23.8 \%$ at age 16 and $29.2 \%$ at age 17 . Except for illicit drugs, differences by age group seem to be less pronounced between ages 16 and 17. For cannabis use, increase in cumulative use rates was strongest between ages $14(5.4 \%)$ and 15 (20.8\%). For alcohol use (67.7-86.3\%) and nicotine use (56.2-71.2\%), increases between ages 14 and 15 were less pronounced. 


\subsubsection{Baseline findings for SUD.}

At baseline rates were $12.5 \%$ for nicotine, $7.4 \%$ for alcohol and $2.6 \%$ for CUD. Rates for alcohol, nicotine and other illicit substance disorders differed slightly between age groups, with slightly higher rates in each successive age cohort considered. Increase of SUD of other illicit substances was strong between age cohort 16 and 17. For CUD, a pronounced increase was found between age groups $14(0.6 \%)$ and $15(3.2 \%)$, while rates remained relatively stable from age groups 15 to $16(3.1 \%)$ and $17(3.9 \%)$.

\subsubsection{Cumulative incidence at $\mathrm{t} 3$.}

Rates (across all age groups) for use as well as for use disorders increase from baseline to final follow-up 10 years later. Cumulative incidence rates for use increased to values of $95.1 \%$ for alcohol and of $54.3 \%$ for cannabis. Rates for use disorders increased to a high of $28.2 \%$ for nicotine, to $27.8 \%$ for alcohol and to $13.7 \%$ for CUD. The increase in rates for CU (19.3\% prevalence at to and $54.3 \%$ cumulative incidence at $\mathrm{t} 3)$ and use of other illicit substances ( $4.8 \%$ at $\mathrm{t} 0$ and $19.7 \%$ at $\mathrm{t} 3$ ) was especially pronounced, the differences for alcohol $(85.7 \%$ at $\mathrm{t} 0$ and $95.1 \%$ at $\mathrm{t} 3)$ and nicotine use $(68.6 \%$ at $\mathrm{t} 0$ and $75.6 \%$ at $\mathrm{t} 3)$ were relatively small. The differences in rates for CUD between t0 and $\mathrm{t} 3$ was especially pronounced for CUD (2.6\% prevalence at t0 versus $13.7 \%$ cumulative incidence at $\mathrm{t} 3)$ and for other illicit substances $(0.5 \%$ at $\mathrm{t} 0$ and $3.5 \%$ at $\mathrm{t} 3)$.

At $\mathrm{t} 3$, differences in rates between age groups were minor for use and disorders of all substances, except for indications of higher rates of alcohol use disorder $(23.5 \%$ in the youngest group versus $31.8 \%$ in the oldest group) and other illicit drug use disorders, respectively $(3.2 \%$ versus $5.1 \%)$.

\subsection{Cross-sectional associations between mental disorders and CU and use disorder at baseline}

To examine the cross-sectional association of a wide range of baseline mental disorders with $\mathrm{CU}$ and CUDs, Table 3 reports the prevalence of mental disorders at baseline and the frequency of each mental disorder by CU and CUD along with their association (odds ratio and 95\% CI). Substance use disorders were included in the list, except for CU and CUD. At baseline $46.2 \%$ of all $14-17$ years old met or had met during the previous life course criteria for at least one lifetime diagnoses listed, almost every second case met criteria for more than one disorder. Most frequent conditions were anxiety disorders $(22.8 \%)$, substance use disorders $(18.1 \%)$, and mood disorders $(11.2 \%)$. Conduct problems were also reported frequently $(24.3 \%)$. Controlling for gender, the cross-sectional analysis revealed that CU was associated with having any mental disorder (OR: 3.1; CI: 2.3-4.0), any mood disorder (OR: 3.5; CI: 2.3-5.2), any anxiety disorder (OR: 1.7; CI: 1.2-2.3), other substance use disorders (OR ranged from 6.2 to 10.9) as well as with ADHD (OR: 2.1; CI: 1.0-4.1) and a middle (OR: 1.9; CI: 1.0-3.4) or high degree (OR: 2.2; CI: 1.3-3.8) of conduct problems in childhood. Among mood disorders, the odds for major depression (OR: 2.7; CI: 1.6-4.4), dysthymia (OR: 6.0; CI: 2.6-13.8) and hypomania/mania (OR: 4.7; CI: 2.2-10.0) are noteworthy. Among anxiety disorders, the odds for panic-anxiety (OR: 5.2; CI: 2.6-10.2) were remarkable; another association was found for separation anxiety disorder (OR: 2.3 ; CI: $1.2-4.3)$.

Associations for CUD were similar, however attenuated, probably due to lower base rates for CUDs. For ADHD, ODD and conduct problems, associations were not significant anymore. Table 3 also revealed a considerable degree of comorbidity among CUD-cases. There were only 10 people $(18.4 \%)$ with CUD that had no co-morbid diagnosis and the majority of cases 
with CUD (59.3\%) had at least one other substance use disorder. Furthermore, the number of comorbid diagnoses had an effect on the prevalence of CU and CUD. The probability of subjects with three or more comorbid diagnoses for CU was $69.6 \%$ (OR: 8.3, CI: 5.3-12.8) and for CUD 32.9\% (OR: 12.1, CI: 5.1-28.4).

\subsection{Prospective associations}

The prospective analyses between baseline mental disorders and incident CU and CUD, respectively (Table 4) confirmed by and large the relationship observed in the cross-sectional analysis, with few exceptions. Having any disorder at baseline was associated with increased rates of CU (OR: 2.1; CI: 1.6-2.7) and CUD (OR: 2.4; CI: 1.6-3.5). Having comorbid disorders was significantly associated with increased rates of CU and CUD; associations were most distinct for having three or more comorbid disorders.

Any substance use disorder, any mood disorder and any anxiety disorder were associated with CU. Among mood disorders, major depression (OR/CU: 1.9; OR/CUD: 2.5) and hypomania/ mania (OR/CU: 2.5; OR/CUD: 2.7) were associated with incident CU and CUD. The OR for dysthymia (OR/CU: 4.0) and CUD was attenuated due to low base rates and was not significant. Among anxiety disorders, only panic-anxiety was associated with CU (OR: 3.5; CI: 1.6-7.3), while for CUD significant associations were found for specific phobias (OR: 1.8; CI: 1.1-2.9) and for GAD(OR: 3.9; CI: 1.1-13.7). For externalizing disorders a high degree of conduct problems was a significant predictor for CU (OR: 2.3, CI: 1.2-4.2); it is noteworthy, though, that for CUD none of the comparisons was significant.

\subsection{Age of onset characteristics for use and dependence}

To describe the age of onset characteristics of CU and CUD by prior history of major depression, bipolar disorder, as well as panic-anxiety, conduct problems and ADHD, a series of Cox proportional hazard analyses were run, to describe and confirm in greater detail these associations controlling for age and gender, taking subjects without the respective diagnoses as a reference. In agreement with the previous analyses, prior history of panic-anxiety predicted CU $(\mathrm{p}<.000)$ incidence. A history of major depression predicted subsequent $\mathrm{CU}$ onset $(\mathrm{p}=.006)$ and CUD $(\mathrm{p}=.003)$. For a history of conduct problems, only a higher level of conduct problems (three or more symptoms reported) predicted CU $(p=.001)$, for CUD coxproportional hazard assumptions were not met. A history of ADHD predicted CU $(p=.031)$, but not CUD. Prior history of bipolar disorder predicted the increased onset of CUD ( $p=$ .020), but not of CU.

\subsection{Effects of panic anxiety, bipolar and depressive symptoms controlling for externalizing disorders, age, and gender}

A series of multiple logistic regressions were run to test, whether the significant effects for specific mental disorders (depressive, bipolar and panic-anxiety) in the prospective model for cannabis use and disorders remain significant after controlling for the presence of externalizing disorders as well as age and gender effects. After controlling for externalizing disorders (conduct disorder, ODD and ADHD), age, and gender, the associations between major depression/dysthymia and CU (OR: 2.2; CI: 1.3-3.7), between hypomania/mania and CU (OR: 2.4; CI: 1.0-5.4) and between panic-anxiety and CU (OR: 3.1; CI: 1.4-6.8) remained significant. For CUD, the associations between major depression/dysthymia and CUD (OR: 2.4; CI: 1.3-4.5) as well as between hypomania/mania and CUD (OR: 2.7; CI: 
1.1-6.3) were significant, while the associations with panic-anxiety was attenuated and not significant anymore.

\section{Discussion}

The first purpose of this paper was to inform about the cumulative incidence of substance use and substance use disorder with an emphasis on cannabis use and cannabis use disorders in adolescents, aged 14-17 at baseline of the EDSP study over a period of 10 years. In agreement with previous publications (Holly and Wittchen, 1998; Lieb et al., 1998; Nelson and Wittchen, 1998; Perkonigg et al., 1998, 1999; von Sydow et al., 2001, 2002a,b;

Zimmermann et al., 2003, 2005) the 10 years data highlight very high rates of use and abuse for all types of substances considered as well as the differential temporal dynamic of various substances. Virtually all subjects have been exposed to alcohol at some time during their life and almost two-thirds to nicotine. Exposure rates to cannabis were particularly high with more than $50 \%$ of the sample having at least tried cannabis in their lifetime. With regard to the temporal order of use the results indicate that first use of alcohol and nicotine occurs quite early in this sample. By the age of 13 for alcohol, and 14 for nicotine, the majority of all adolescents had used licit drugs at least once. In the majority of subjects, regular use occurred only slightly later (1.3 years; not reported in this paper, see Perkonigg et al., 2006).

Concerning cumulative incidence of CU and CUD as the most frequent illicit drug of abuse, several things are notable: at baseline, the measures for CU for the youngest cohort (aged 14) were lower than for the three older cohorts. This may lead to the conclusion that use of cannabis starts at about age 15. Age of onset analysis revealed, that CU even started before age 15 for a significant proportion of individuals.

While there is a strong increase in rates for use of cannabis between baseline and the final follow-up examination, the same difference is not found for nicotine and alcohol. This may lead to the conclusion, that the first use of cannabis takes place later in adolescence, while first use of legal substances like alcohol and nicotine takes place earlier. The result may be due to relatively liberal attitudes toward use of nicotine and alcohol (legal age of use of nicotine and alcohol is 16 years; very little restrictive legal regulations and few enforcement) in Germany. It might be of interest to examine whether this age of onset pattern is different in other populations with more restrictive policies.

At baseline, the measures for CU for age cohorts 15,16 , and 17 ranged from $20.8 \%$ to $29.2 \%$. At the final follow-up measures for all age cohorts ranged from $51.1 \%$ to $54.5 \%$. Thus, numbers of first use may increase substantially between age 18 and 23. For CUD, rates for all age groups increase from baseline to final follow-up. While rates for alcohol abuse/dependence and nicotine dependence also increase, the differences between baseline and final follow-up is clearly more pronounced for CUD (2.6\% at baseline; $13.7 \%$ at final follow-up). Overall, this might indicate, that first incidence of CUD tends to occur later than nicotine dependence and alcohol abuse/dependence. Yet, the difference between cannabis and alcohol resp. nicotine concerning the differences between baseline and final follow-up is not as distinct for CUD as for CU. At baseline, the measures for CUD for age cohorts 15, 16, and 17 ranged from $3.2 \%$ to $3.9 \%$; at final follow-up measures for all age cohorts ranged from $11.5 \%$ to $16.5 \%$. Thus, numbers of CUD may increase between age 18 and 23 . For the two older age cohorts, increase of CUD cumulative incidence rates between baseline and final follow-up was not as large as for the two younger cohorts, possibly indicating the existence of a cohort effect that does not appear at baseline. 
Onset for first CU occurred on average around the age of 15 , slightly before first use of other illicit drugs. Among substance use disorders nicotine and alcohol were the most prevalent with onset for abuse and dependence almost always occurring before the age of 18 . The findings of high prevalence and high rates of concomitant substance use are by and large consistent with previous literature, although the early onset characteristics are remarkable and have important prevention and public health implications (Hawkins et al., 1992; Wittchen, 2004). In short, one of the major public health goals for Germany should be a difference of age of onset of substance use, as this indicator has often been linked to problematic consequences and considerable costs (specifically for tobacco: Vega and Gil, 2005; other drugs: Hawkins et al., 1992; general considerations: Stockwell et al., 2005), even if these problematic consequences are reported from observations of US samples.

The second goal of this paper was to describe more comprehensively than previous studies the association of CU and CUD with a wider range of mental disorders. Beyond the well established associations with externalizing disorders as well as preceding other substance use disorders (e.g. effects of nicotine or alcohol dependence on CU and disorders) that were largely confirmed in the present analyses, our findings reveal:

(a) Cross-sectional associations, and weaker, but still meaningful prospective associations with depressive, bipolar and some anxiety disorders.

(b) Most consistent associations were found for all mood disorders, including bipolar disorders (hypomania and mania), that also predicted increased rates for CU and CUD in the prospective model (except dysthymia, that did not predict CUD).

(c) Among anxiety disorders, associations with specific disorders were variable. Relatively consistently, panic-anxiety was associated with CU and CUD and predicted CU in the prospective model. History of separation anxiety disorder was associated with CU and CUD, but not significant as a predictor. In the prospective model, GAD and specific phobias were associated with CUD, but not with CU. GAD and specific phobias were not associated with CU/CUD in the cross-sectional analysis. These findings seem to be consistent with the observation, that in the first decades of life, anxiety disorders have a relatively low stability (Wittchen et al., 2000).

(d) The prospective associations for mood and anxiety disorders with CU and CUD seem to be similar, suggesting that the role of previous mental disorders might not be that different in initiating first use, developing regular use and developing dependence. Yet, notable exceptions might be dysthymia, panic-anxiety, and conduct problems, that predict only CU.

(e) Using Cox proportional hazard modelling, taking into account mental disorders throughout the complete follow-up period, revealed a significant relationship with panic-anxiety for $\mathrm{CU}$, with bipolar disorders for CUD and with major depression for CU and CUD. A history of ADHD as well as the highest degree of conduct problems predicted CU, but not CUD.

(f) After controlling for externalizing disorders (ADHD, ODD, CD), depressive disorders, and hypomania/mania predicted CU as well as CUD while panic-anxiety predicted only CU.

(g) No significant associations of mental disorders with CU and CUD were found for PTSD. It should be noted, that only few cases met criteria for PTSD. 
(h) Among externalizing disorders no consistent findings were found for ODD and ADHD as predictors for CU and CUD.

To summarize, for associations and prospective associations with CU/CUD, overall, no homogeneous pattern could be found for the role of internalizing disorders examined in this paper at least with regard to the prediction of CU and CUD. Results reveal that mood disorders, both of uni- and bipolar form, play a significant predictor role for increased rates of $\mathrm{CU}$ and CUD, whereas the effects for anxiety disorders appeared to be variable. Some anxiety disorders may be of relevance for predicting increased rates, but were not frequent enough to verify an effect. The only consistent and strong effect was observed for panic-anxiety. However, panic-anxiety did not predict CUD and it needs to be studied further, whether this effect is indicative for specific anxiety disorders. It may also be that this finding corroborates results from other studies that have suggested panic anxiety might be a diagnostically unspecific risk marker for a whole range of psychopathologies (Goodwin et al., 2004), playing a significant role only in the initiation of use. This would be consistent with the observation that most of those using cannabis also use nicotine. Associations between nicotine and panic have already been discussed by Isensee et al. (2003).

The fact, that associations with separation anxiety could be observed at baseline but not at final follow-up, as well as the fact, that associations with GAD and specific phobias and CUD were found at final follow-up but not at baseline may indicate, that specific disorders may influence CU and CUD at different points of time in adolescence and young adulthood. For GAD, it may be assumed that the influence of GAD on CUD takes place later in adolescence, since GAD itself tends to occur later in adolescence. For specific phobias, the results remain surprising because specific phobias tend to occur in childhood. Yet, subtypes of specific phobias tend to occur later and may have contributed to the results observed here. A further investigation of the associations of GAD and subtypes of specific phobias might be useful.

It might also be useful to investigate why panic-anxiety and dysthymia were associated to CU and CUD and predicted CU but not CUD. These results may indicate, that the influence of these disorders on the development of CUD is of greater importance earlier in adolescence.

The question of the specificity, the role of comorbid patterns and the implications of anxiety and depressive disorders and early childhood conditions on the risk for CU and CUD were not dealt with in this paper. However, it seems to be evident, that a wide range of psychopathological conditions, beyond externalising disorders plays a significant, but yet poorly understood role. The longitudinal interplay between early childhood and adolescent anxiety, depressive, and substance use and disorders other than cannabis (usually lumped together in the term comorbidity) clearly deserves future research attention. The most pronounced associations between mental disorders and CU and CUD were found for those with multiple disorders, both in predicting CU and CUD. Further analyses will focus on identifying potential syndrome specific effects in contrast to a joint diathesis of psychopathology in predicting use and the transitions to disorders.

With regard to externalizing disorders our findings confirm the role of conduct disorders for increased rates of use, but less so for use disorders. It is also noteworthy, that for ADHD and ODD findings were not as consistent as for conduct problems. This indirectly confirms observations by Fergusson et al. (in this edition), who suggested that elevated substance rates among ADHD cases might be the consequence of comorbid conduct problems rather than a consequence of ADHD as suggested from a clinical perspective (Horner and Scheibe, 1997). 
The third goal of this paper was to examine whether internalizing disorders are associated with CU/CUD beyond the effects of externalizing disorders. The results found when controlling for externalizing disorders (ADHD, ODD, CD) suggest that depressive disorders and hypomania/mania independently predict CU and CUD while panic-anxiety independently predicts CU.

In further analyses the developmental psychopathological perspective between early childhood syndromes and adolescent mood and anxiety disorders need to be examined more closely, focusing specifically on the co-variation of use of various substances. Before the onset of CUD, a large proportion experienced other mental or substance use disorder. For CUD, the exact proportion was $53.7 \%$. $68.8 \%$ of the people with mental or substance use disorders prior to a CUD had an alcohol and $63.4 \%$ had a nicotine use disorder. For other illicit drug use disorders, in addition, CUD is a powerful predictor with an OR of 24.4 (CI: 10.1-58.8). Among several explanations, one potential causal mechanism to explain this covariation may be that cannabinoids can induce behavioral sensitization and cross-sensitization to other drugs, especially opioids (Arnold, 2005; Cadoni et al., 2001; Lamarque et al., 2001). However, this speculation and hypothesis needs further testing.
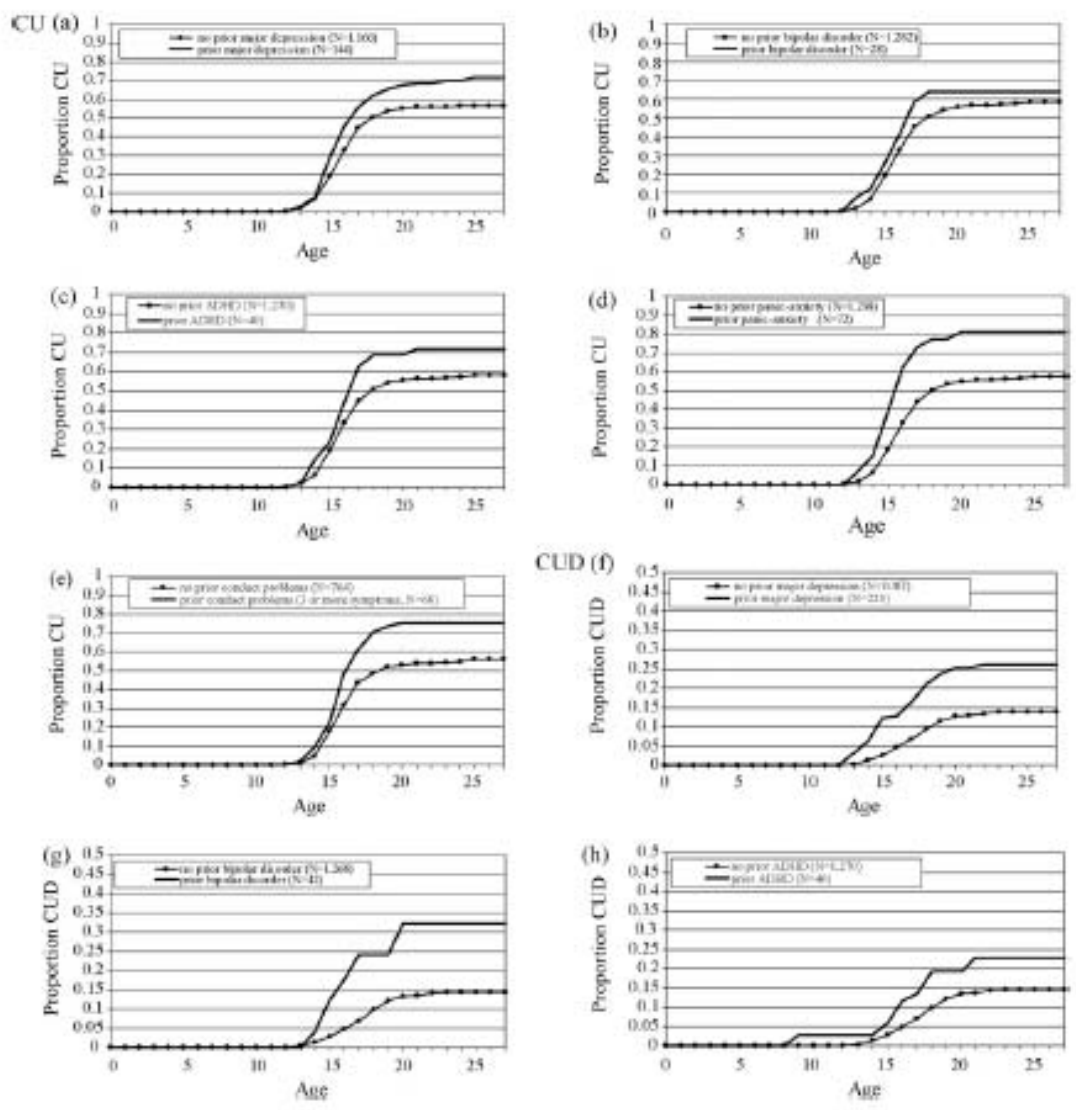

Fig. 1. Cumulative incidence of cannabis use (a-e) and cannabis use disonders ( $\mathrm{f}-\mathrm{h}$ ) by prior history of major depression, bipolar disorder, panic-anxiety, ADHD, and conduct problems. Cox proportional hazard models stratified for age and gender. For the associations between panic-anxiety and cannabis use disonder as well as between conduct problems and cannabis use disorder, no figures are displayed because the cox-proportional hazard assumption was not met in these two cases. 
Table 1

Prospective epidemiological studies on the association of cannabis use/use disorder and mental disorders in 14-18 years old adolescents

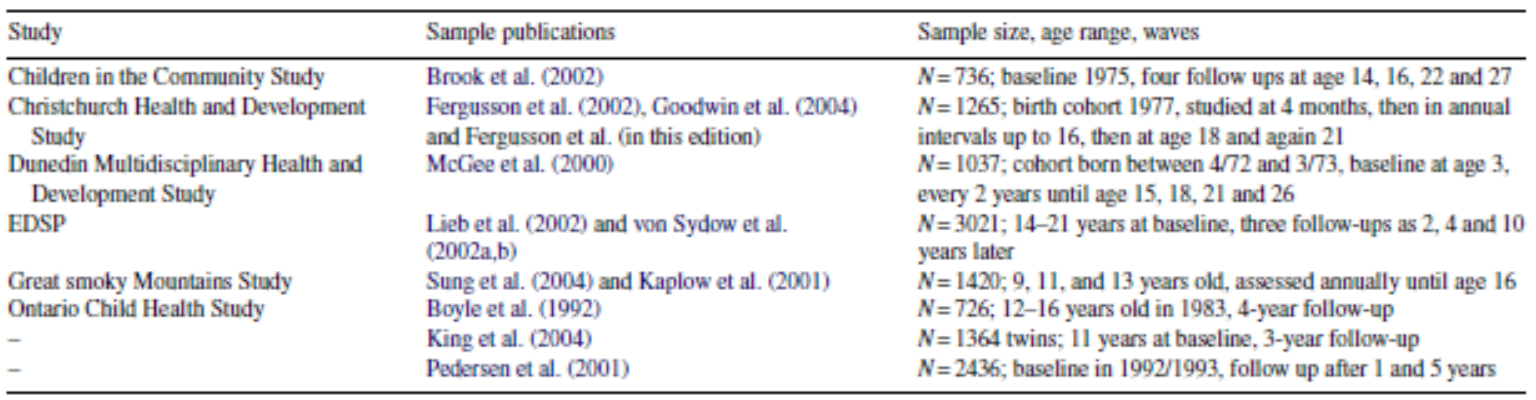

Table 2

Prevalence and cumulative incidence of substance use and substance use disorders among individuals aged 14-17 at the outset of the EDSP study

\begin{tabular}{|c|c|c|c|c|c|c|c|c|}
\hline & \multicolumn{4}{|c|}{ Baseline prevalence $(N=1395)$} & \multicolumn{4}{|c|}{ Cumulative incidence T3 $(N=1395)$} \\
\hline & $N$ & $\% w^{b}$ & $N$ & $\% w^{b}$ & $N$ & $\% w^{b}$ & $N$ & $\approx w^{b}$ \\
\hline \multicolumn{9}{|l|}{ Total $(N=1395)$} \\
\hline Alcohol & 1160 & 85.66 & 82 & 7.44 & 1321 & 95.10 & 380 & 27.81 \\
\hline Nicotine & 937 & 68.60 & 150 & 12.50 & 1049 & 75.64 & 388 & 28.16 \\
\hline Cannabis $^{c, d}$ & 233 & 19.33 & 33 & 2.61 & 706 & 54.29 & 131 & 13.69 \\
\hline \multicolumn{9}{|c|}{14 years at $T 0(N=498)$} \\
\hline Aloohol & 341 & 67.72 & 4 & 0.76 & 456 & 90.86 & 120 & 23.49 \\
\hline Nicotine & 283 & 56.18 & 15 & 3.29 & 354 & 70.88 & 116 & 23.46 \\
\hline Cannabis c,d & 28 & 5.40 & 3 & 0.56 & 237 & 51.09 & 52 & 14.78 \\
\hline Other ill. drugs $s^{\varepsilon, d}$ & 7 & 1.47 & 0 & & 82 & 17.29 & 15 & 3.17 \\
\hline Any substance $e^{c, d}$ & 374 & 78.86 & 21 & 4.42 & 445 & 96.19 & 183 & 39.10 \\
\hline Cannabis $^{c, d}$ & 81 & 20.75 & 13 & 3.21 & 217 & 56.96 & 44 & 16.47 \\
\hline Other ill drugse,d & 17 & 4.30 & 1 & 0.22 & 76 & 19.67 & 9 & 2.25 \\
\hline Any substance $e^{c, d}$ & 355 & 90.34 & 62 & 16 & 374 & 97.01 & 185 & 47.95 \\
\hline \multicolumn{9}{|c|}{16 years at $\mathrm{TO}(N=271)$} \\
\hline Alcohol & 255 & 93.80 & 29 & 10.10 & 262 & 96.38 & 82 & 29.10 \\
\hline Nicotine & 199 & 72.08 & 47 & 18.10 & 212 & 76.39 & 84 & 30.11 \\
\hline Cannabis ${ }^{c, d}$ & 64 & 23.76 & 9 & 3.05 & 143 & 55.14 & 21 & 11.74 \\
\hline Other ill. drugse,d & 10 & 3.85 & 1 & 0.51 & 50 & 19.84 & 9 & 3.62 \\
\hline Any substance $e^{c, d}$ & 254 & 95.98 & 67 & 25.40 & 249 & 97.07 & 126 & 48.67 \\
\hline \multicolumn{9}{|c|}{17 years at $\mathrm{TO}(N=220)$} \\
\hline Alcohol & 214 & 97.62 & 33 & 15.50 & 216 & 98.50 & 68 & 31.75 \\
\hline Nicotine & 168 & 76.93 & 36 & 17.10 & 172 & 79.15 & 65 & 29.51 \\
\hline
\end{tabular}

a Abuse and dependence.

b Percents weighted.

c 55 respondents refused to answer to illicit drug questions at baseline.

d 85 respondents refused to answer to illicit drug questions at any wave. 
Dahic 3

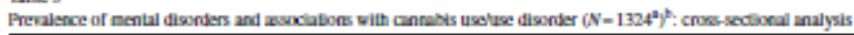

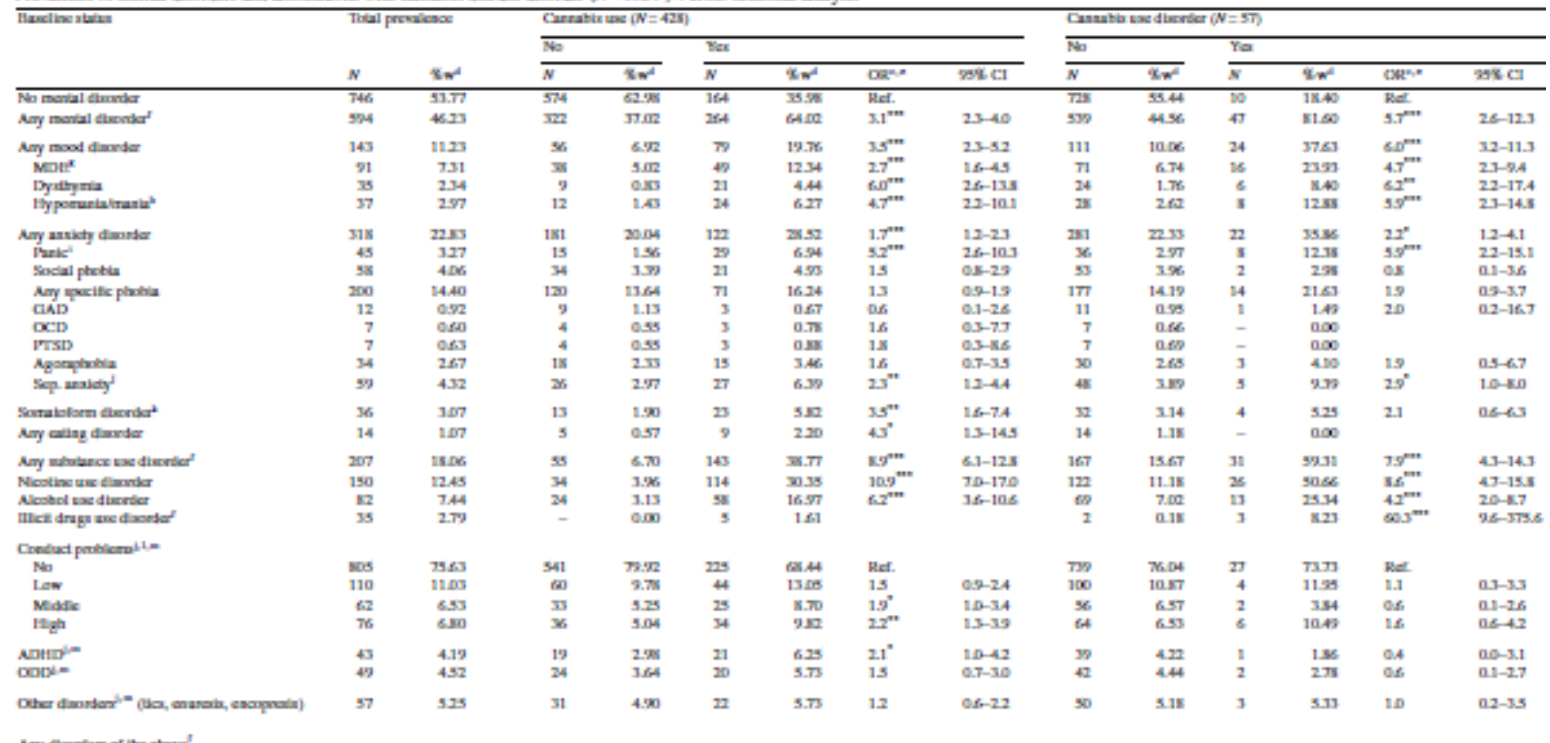

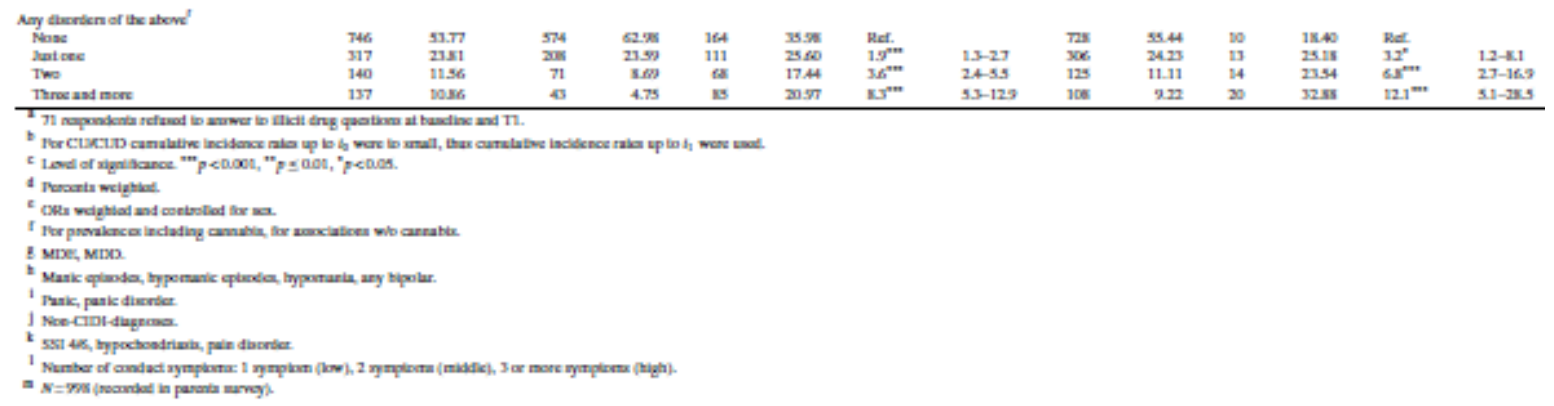


Table 4

Cumulative lifetime incidence of cannabis use and cannabis use disorder by baseline mental disorders $\left(N=1310^{2}\right)$ : prospective analysis

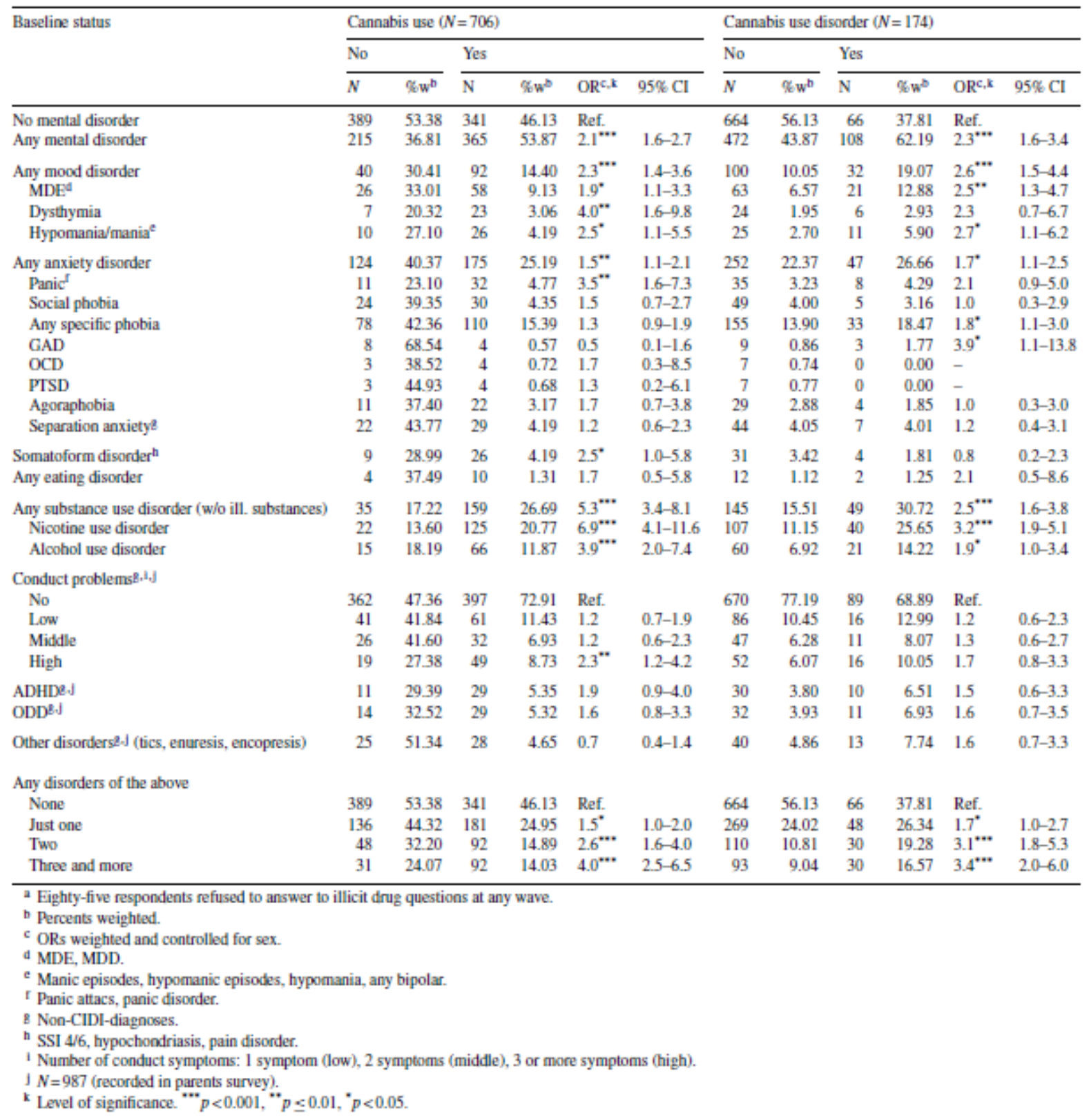

\section{Acknowledgements}

This paper has been prepared in the context of the project F1 "Community-based need evaluation II \& Allocation and transfer" (PI: H.U. Wittchen) of the Addiction Research Network ASAT (Allocating Substance Abuse Treatments to Patient Heterogeneity). Contact information: e-mail: asatkoordination@mpipsykl.mpg.de (www.asatverbund. de).ASAT is sponsored by a federal grant of the Federal Ministry of Education and Research (01 EB 04400442, 01 EB 0142).

This work is further part of the Early Developmental Stages of Psychopathology (EDSP) Study and is funded by the German Federal Ministry of Education and Research (BMBF) project no. 01EB9405/6, 01EB 9901/6, EB01016200, 01EB0140, and 01EB0440. Part of the field work and analyses were also additionally supported by grants of the Deutsche Forschungsgemeinschaft (DFG) LA1148/1-1, WI2246/1-1, WI 709/7-1, and WI 709/8-1. 
Furthermore, this study is part of the "Multi-site international comparison of unmet needs" andwas supported by a grant from the National Institute of Health (NIH; 303-7028; PI: Jane Costello).

Principal investigators of the EDSP are Dr. Hans-Ulrich Wittchen and Dr. Roselind Lieb. Core staff members of the EDSP group are: Dr. Kirsten von Sydow, Dr. Gabriele Lachner, Dr. Axel Perkonigg, Dr. Peter Schuster, Dipl.-Stat. Michael Höfler, Dipl.-Psych. Holger Sonntag, Dipl.-Psych. Tanja Brückl, Dipl.-Psych. Elzbieta Garczynski, Dr. Barbara Isensee, Dipl.-Psych. Agnes Nocon, Dr. Chris Nelson, Dipl.-Inf. Hildegard Pfister, Dr. Victoria Reed, Dipl.-Soz. Barbara Spiegel, Dr. Andrea Schreier, Dr. Ursula Wunderlich, Dr. Petra Zimmermann, Dr. Katja Beesdo and Dipl.-Psych. Antje Bittner. Scientific advisors are Dr. Jules Angst (Zurich), Dr. Jürgen Margraf (Basel), Dr. Günther Esser (Potsdam), Dr. Kathleen Merikangas (NIMH, Bethesda), Dr. Ron Kessler (Harvard, Boston) and Dr. Jim van Os (Maastricht).

\section{References}

- Agresti, A., 1990. Categorical Data Analysis. John Wiley \& Sons, New York.

- Arnold, J.C., 2005. The role of endocannabinoid transmission in cocaine addiction. Pharmacol. Biochem. Behav. 81, 396-406 (special issue: Cannabinoids: Interactions with other drugs).

- $\quad$ Boyle, M.H., Offord, D.R., Racine, Y.A., Szatmari, P., Fleming, J.E., Links, P.S., 1992. Predicting substance use in late adolescence - results from the Ontario child health study follow-up. Am. J. Psychiatry 149, 761-767.

- Brook, D.W., Brook, J.S., Zhang, C.S., Cohen, P., Whiteman, M., 2002. Drug use and the risk of major depressive disorder, alcohol dependence, and substance use disorders. Arch. Gen. Psychiatry 59, 1039-1044.

- Cadoni, C., Pisanu, A., Solinas, M., 2001. Behavioural sensitization after repeated exposure to $\Delta$-sup-9-tetrahydrocannabinol and cross-sensitization with morphine. Psychopharmacology 158, 259-266.

- Fergusson, D.M., Horwood, L.J., Swain-Campbell, N., 2002. Cannabis use and psychosocial adjustment in adolescence and young adulthood. Addiction 97, 1123-1135.

- Fergusson, D.M., Horwood, L.J., Ridder, E.M., 2007. Conduct and attentional problems in childhood and adolescence and later substance use, abuse and dependence. Drug Alcohol Depend 88S, S14-S26.

- Goodwin, R.D., Fergusson, D.M., Horwood, L.J., 2004. Association between anxiety disorders and substance use disorders among young persons: results of a 21-year longitudinal study. J. Psychiatr. Res. 38, 295-304.

- Grambsch, P.M., Therneau, T.M., 1994. Proportional hazards tests and diagnostics based on weighted residuals. Biometrika 81, 515-526.

- Hawkins, J.D., Catalano, R.F., Miller, J.Y., 1992. Risk and protective factors for alcohol and other drug problems in adolescence and early adulthood: implications for substance abuse prevention. Psychol. Bull. 112 (1), 64-105.

- Henquet, C., Krabbendam, L., Spauwen, J., Kaplan, C., Lieb, R., Wittchen, H.-U., van Os, J., 2005. Prospective cohort study of cannabis use, predisposition for psychosis, and psychotic symptoms in young people. Br. Med. J. 331, 11-14.

- Höfler, M., Lieb, R., Perkonigg, A., Schuster, P., Sonntag, H., Wittchen, H.-U., 1999. Covariates of cannabis use progression in a representative population sample of adolescents: a prospective examination of vulnerability and risk factors. Addiction 94, 1679-1694.

- Holly, A., Wittchen, H.-U., 1998. Patterns of use and their relationship to DSMIV abuse and dependence of alcohol among adolescents and young adults. Eur. Addict. Res. 4, 50-57.

- Horner, B.R., Scheibe, K.E., 1997. Prevalence and implications of attention-deficit hyperactivity disorder among adolescents in treatment for substance abuse. J. Am. Acad. Child Adolesc. Psychiatry 36, 30-36. 
- Isensee, B., Wittchen, H.-U., Stein, M.B., Höfler, M., Lieb, R., 2003. Smoking increases the risk of panic. Findings from a prospective community study. Arch. Gen. Psychiatry 60, 692 700 .

- Kaplow, J.B., Curran, P.J., Angold, A., Costello, E.J., 2001. The prospective relation between dimensions of anxiety and the initiation of adolescent alcohol use. J. Clin. Psychol. 30, 316326.

- $\quad$ King, S.M., Iacono, W.G., McGue, M., 2004. Childhood externalizing and internalizing psychopathology in the prediction of early substance use. Addiction 99, 1548-1559.

- Lachner, G., Wittchen, H.-U., Perkonigg, A., Holly, A., Schuster, P., Wunderlich, U., Türk, D., Garczynski, E., Pfister, H., 1998. Structure, content and reliability of the Munich Composite International Diagnostic Interview (M-CIDI), substance use sections. Eur. Addict. Res. 4, 28-41.

- $\quad$ Lamarque, S., Taghzouti, K., Simon, H., 2001. Chronic treatment with $\Delta$-sup-9tretrahydrocannabinol enhances the locomotor response to amphetamine and heroin. Implications for vulnerability to drug addiction. Neuropharmacology 41, 118-129.

- Lieb, R., Isensee, B., von Sydow, K., Wittchen, H.-U., 2000. The early developmental stages of psychopathology study (EDSP): a methodological update. Eur. Addict. Res. 6, 170-182.

- $\quad$ Lieb, R., Pfister, H., Wittchen, H.-U., 1998. Use, abuse and dependence of prescription drugs in adolescents and young adults. Eur. Addict. Res. 4, 67-74.

- $\quad$ Lieb, R., Schuetz, C.G., Pfister, H., von Sydow, K., Wittchen, H.-U., 2002. Mental disorders in ecstasy users: a prospective-longitudinal investigation. Drug Alcohol Depend. 68, 195-207.

- Long, J.S., Freese, J., 2003. Regression Models for Categorical Dependent Variables Using Stata. Stata Press, College Station, TX.

- McGee, R., Williams, S., Poulton, R., Moffitt, T., 2000. A longitudinal study of cannabis use and mental health from adolescence to early adulthood. Addiction 95, 491-503.

- Nelson, C.B., Wittchen, H.-U., 1998. Smoking and nicotine dependence: results from a sample of 14-24 years old in Germany. Eur. Addict. Res. 4, 42-49.

- Pedersen, W., Mastekaasa, A., Wichstrom, L., 2001. Conduct problems and early cannabis initiation: a longitudinal study of gender differences. Addiction 96, 415-431.

- Perkonigg, A., Lieb, R., Wittchen, H.-U., 1998. Prevalence of use, abuse and dependence of illicit drugs among adolescents and young adults in a community sample. Eur. Addict. Res. 4, 58-66.

- Perkonigg, A., Lieb, R., Höfler, M., Schuster, P., Sonntag, H., Wittchen, H.-U., 1999. Patterns of cannabis use, abuse and dependence over time: incidence, progression and stability in a sample of 1228 adolescents. Addiction 94, 1663-1678.

- Perkonigg, A., Pfister, H., Höfler, M., Fröhlich, C., Zimmermann, P., Lieb, R., Wittchen, H.U., 2006. Substance use and substance use disorders in a community sample of adolescents and young adults: incidence, age effects and patterns of use. Eur. Addict. Res. 12, 187-196.

- $\quad$ Reed, V., Gander, F., Pfister, H., Steiger, A., Sonntag, H., Trenkwalder, C., Sonntag, A., Hundt, W., Wittchen, H.-U., 1998. To what degree the Composite International Diagnostic Interview (CIDI) correctly identify DSM-IV disorders? Testing validity issues in a clinical sample. Int. J. Meth. Psychiatr. Res. 7, 142-155.

- Royall, R.M., 1986. Model robust confidence intervals using maximum likelihood estimators. Int. Stat. Rev. 54, 221-226.

- StataCorp., 2003. Stata Statistical Software: Release 8.0 (Version College-Station). Stata Corporation, TX.

- Stockwell, T., Gruenewald, P., Toumbourou, J., Loxley, W. (Eds.), 2005. Preventing Harmful Substance Use: The Evidence Base for Policy and Practice. John Wiley \& Sons.

- Sung, M., Erkanli, A., Angold, A., Costello, E.J., 2004. Effects of age at first substance use and psychiatric comorbidity on the development of substance use disorders. Drug Alcohol Depend. 75, 287-299.

- Therneau, T.M., Grambsch, P.M., 2000. Modeling Survival Data: Extending the Cox Model. Springer, New York.

- $\quad$ von Sydow, K., Lieb, R., Pfister, H., Höfler, M., Wittchen, H.-U., 2002a. What predicts incident use of cannabis and progression to abuse or dependence? A 4-year prospective 
examination of risk factors in a community sample of adolescents and young adults. Drug Alcohol Depend. 68, 49-64.

- $\quad$ von Sydow, K., Lieb, R., Pfister, H., Höfler, M., Wittchen, H.-U., 2002b. Use, abuse and dependence of ecstasy and related drugs in adolescents and young adults - a transient phenomenon? Results from a longitudinal community study. Drug Alcohol Depend. 66, 147159.

- $\quad$ von Sydow, K., Lieb, R., Pfister, H., Höfler, M., Sonntag, H., Wittchen, H.-U., 2001. The natural course of cannabis use, abuse and dependence over 4 years: a longitudinal community study of adolescents and young adults. Drug Alcohol Depend. 64, 347-361.

- Vega, W.A., Gil, A.G., 2005. Revisiting drug progression: long-range effects of early tobacco use. Addiction 100 (9), 1358-1369.

- Wittchen, H.-U., Pfister, H., 1997. DIA-X-Interview: Instruktionsmanual zur Durchführung von DIA-X-Interviews. Swets \& Zeitlinger, Frankfurt.

- Wittchen, H.-U., 1994. Reliability and validity studies of the WHO-Composite International Diagnostic Interview (CIDI): a critical review. J. Psychiatr. Res. 28, 57-84.

- Wittchen, H.-U., Beloch, E., Garczynski, E., Holly, A., Lachner, G., Perkonigg, A., Pfütze, E.M., Schuster, P., Vodermaier, A., Vossen, A., Wunderlich, U., Zieglgänsberger, S., 1995. Münchener Composite International Diagnostic Interview (M-CIDI) (Version 2.2/2/95). MaxPlanck-Institut für Psychiatrie, Klinische Psychologie und Epidemiologie, München.

- Wittchen, H.-U., Brückl, T., Ruhl, U., 2004. Haben frühe psychische Störungen Folgen? In: Lehmkuhl, U., Lehmkuhl, G. (Eds.), Frühe psychische Störungen und ihre Behandlung. Vandenhoeck \& Ruprecht, Göttingen, pp. 25-44.

- Wittchen, H.-U., Lachner, G., Wunderlich, U., Pfister, H., 1998a. Test-retest reliability of the computerized DSM-IV version of the Munich-Composite International Diagnostic Interview (M-CIDI). Soc. Psychiatry Psychiatr. Epidemiol. 33, 568-578.

- Wittchen, H.-U., Nelson, C.B., Lachner, G., 1998b. Prevalence of mental disorders and psychosocial impairments in adolescents and young adults. Psychol. Med. 28, 109-126.

- Wittchen, H.-U., Semler, G., 1991. Composite International Diagnostic Interview (CIDI). Beltz, Weinheim.

- Wittchen, H.-U., Lieb, R., Pfister, H., Schuster, P., 2000. The waxing and waning of mental disorders: evaluating the stability of syndromes of mental disorders in the population. Compr. Psychiatry 41 (2 Suppl. 1), 122-132.

- Zimmermann, P., Wittchen, H.-U. ,Höfler, M., Pfister, H., Kessler, R.C., Lieb, R., 2003. Primary anxiety disorders and the development of subsequent alcohol use disorder: a 4-year community study of adolescents and young adults. Psychol. Med. 33, 1211-1222.

- Zimmermann, P., Wittchen, H.-U., Waszak, F., Nocon, A., Höfler, M., Lieb, R., 2005. Pathways into ecstasy use: impact of cannabis on availability and first use of ecstasy. Drug Alcohol Depend. 79, 331-341. 